\title{
${ }^{123}$ I-MIBG SPECT for Evaluation of Patients with Heart Failure
}

\author{
Aukelien C. Dimitriu-Leen ${ }^{1}$, Arthur J.H.A. Scholte ${ }^{1}$, and Arnold F. Jacobson ${ }^{2}$ \\ ${ }^{I}$ Department of Cardiology, Leiden University Medical Center, Leiden, The Netherlands; and ${ }^{2}$ Diagram Consulting, Kihei, Hawaii
}

\begin{abstract}
Heart failure (HF) is characterized by activation of the sympathetic cardiac nerves. The condition of cardiac sympathetic nerves can be evaluated by ${ }^{123}$-metaiodobenzylguanidine (123/-MIBG) imaging. Most cardiac ${ }^{123}$ |-MIBG studies have relied on measurements from anterior planar images of the chest. However, it has become progressively more common to include SPECT imaging in clinical and research protocols. This review examines recent trends in ${ }^{123}$-MIBG SPECT imaging and evidence that provides the basis for the increased use of the procedure in the clinical management of patients with HF. ${ }^{123}$ I-MIBG SPECT has been shown to be complementary to planar imaging in patients with HF in studies of coronary artery disease after an acute myocardial infarction. Moreover, ${ }^{123}$ /-MIBG SPECT has been used in numerous studies to document regional denervation for arrhythmic event risk assessment. For better quantification of the size and severity of innervation abnormalities in ${ }^{123}$ MIBG SPECT, programs and protocols specifically for ${ }^{123}$ / have been developed. Also, the introduction of new solid-state cameras has created the potential for more rapid SPECT acquisitions or a reduction in radiopharmaceutical activity. Although PET imaging has superior quantitative capabilities, ${ }^{123}$ |-MIBG SPECT is, for the foreseeable future, the only widely available nuclear imaging method for assessing regional myocardial sympathetic innervation.
\end{abstract}

Key Words: ${ }^{123} \mathrm{I}-\mathrm{MIBG}$; SPECT; heart failure

J Nucl Med 2015; 56:25S-30S

DOI: 10.2967/jnumed.115.157503

\section{$\mathbf{H}$}

eart failure (HF) is characterized by activation of the sympathetic cardiac nerves. The condition of cardiac sympathetic nerves can be evaluated with ${ }^{123} \mathrm{I}$-metaiodobenzylguanidine $\left({ }^{123} \mathrm{I}\right.$ MIBG), which is a norepinephrine analog and therefore a tracer for sympathetic neuron integrity and function (1). Most of the literature on the use of ${ }^{123} \mathrm{I}-\mathrm{MIBG}$ imaging for evaluating patients with $\mathrm{HF}$ is based on measurements from anterior planar images of the chest, with cardiac uptake quantified in terms of the heart-to-mediastinum ratio (HMR) and the washout rate between early and late images $(2,3)$. However, given the conversion from planar to SPECT techniques in clinical nuclear myocardial perfusion imaging (MPI) beginning in the 1980s, it is not surprising that ${ }^{123}$ I-MIBG SPECT was also performed by some early adopters of the radiopharmaceutical $(4,5)$. Despite the

\footnotetext{
Received Mar. 13, 2015; revision accepted Apr. 10, 2015.

For correspondence or reprints contact: Arthur J.H.A. Scholte, Department of Cardiology, Leiden University Medical Center, Albinusdreef 2, P.O. Box 9600, 2300 RC Leiden, The Netherlands.

E-mail: a.j.h.a.scholte@lumc.nl

COPYRIGHT (C) 2015 by the Society of Nuclear Medicine and Molecular Imaging, Inc.
}

challenges associated with performing ${ }^{123}$ I-MIBG SPECT and interpreting the images in patients with severely reduced cardiac ${ }^{123}$ I-MIBG uptake, it has become progressively more common for ${ }^{123}$ I-MIBG SPECT imaging to be included in clinical and research protocols; in such settings, it is usually performed as part of one or more imaging sessions in which planar images are acquired (2).

Both qualitative and quantitative image reviews of ${ }^{123} \mathrm{I}-\mathrm{MIBG}$ SPECT studies may be performed to identify focal areas of reduced uptake (similar to protocols used for the interpretation of MPI SPECT studies). Unfortunately, only small amounts of data from ${ }^{123}$ I-MIBG SPECT images of patients without heart disease are available; most often, segmental count density expressed as a percentage of total myocardial count density is used to compare affected areas with least affected areas in patients (6-8). Changes in relative uptake and absolute uptake between early and late SPECT images can be used to calculate parameters comparable to the planar washout rate (9). In general, SPECT results have been used in the same way as planar data-namely, to investigate the relationship between the quantitative value of ${ }^{123}$ I-MIBG uptake and a specific outcome event, such as worsening of chronic HF, the occurrence of an arrhythmic event or cardiac death, or another quantitative parameter, such as a change in the left ventricular ejection fraction or the MPI defect score. Consistent with findings on planar imaging, patients with larger or more severe regional ${ }^{123}$ I-MIBG SPECT defects usually have poorer outcomes (10).

This review examines recent trends in ${ }^{123}$ I-MIBG SPECT imaging and evidence that provides the basis for the increased use of the procedure in the clinical management of patients with HF. The focus of the article is primarily on work that has been done in the past $10-15 \mathrm{y}$, considering specifically how the technique can complement the established value of planar ${ }^{123}$ I-MIBG imaging and provide unique insight into the risk of arrhythmia and the need for advanced therapies. Recent advances in imaging technology and quantitation methods are also briefly reviewed, as these represent the future for the clinical use of cardiac ${ }^{123}$ I-MIBG SPECT.

\section{3|-MIBG SPECT COMPLEMENTS PLANAR IMAGING}

Several studies have examined the use of ${ }^{123}$ I-MIBG imaging as a diagnostic and prognostic tool in patients with $\mathrm{HF}(3,11)$. Although most of these studies relied primarily on planar imaging determinations of HMR and washout rate, some also included measurements from SPECT examinations because of the realization that SPECT may overcome the difficulties of planar imaging, including superposition of noncardiac structures and lack of segmental analysis (Fig. 1). Many such ${ }^{123}$ I-MIBG SPECT studies were performed by Kasama et al., who investigated the effects of a variety of HF medications on ${ }^{123}$ I-MIBG cardiac uptake and clinical outcomes, usually performing imaging before the initiation 


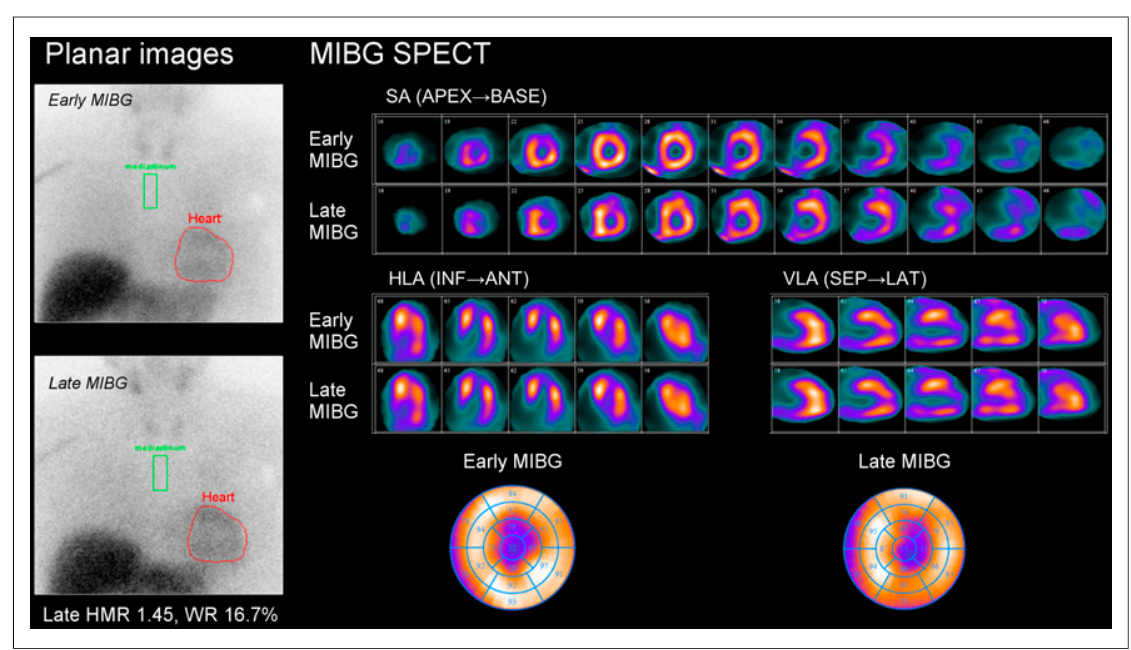

FIGURE 1. ${ }^{123}$-MIBG SPECT complements planar imaging on segmental analysis. Planar images and SPECT images in short axis (SA), horizontal axis (HLA), vertical long axis (VLA), and bull's-eye of ${ }^{123}$ I-MIBG were obtained for 60 -y-old patient. Reduced late HMR was 1.45 . 123I-MIBG SPECT demonstrated fixed apical defect. ANT = anterior; INF = inferior; LAT = lateral; SEP $=$ septal; WR $=$ washout rate.

of a new medication and again after 6 mo (12-19). Early and late ${ }^{123} \mathrm{I}-\mathrm{MIBG}$ SPECT images (acquired at $15 \mathrm{~min}$ and at $4 \mathrm{~h}$ ) were scored visually with 20- and 17-segment regional polar or bull's-eye maps. Additionally, segmental count data were used to calculate regional washout rates in some studies. The various randomized studies demonstrated statistically significant reductions in late ${ }^{123} \mathrm{I}-\mathrm{MIBG}$ total defect scores after treatment with an angiotensin-converting enzyme inhibitor (perindopril), angiotensin receptor blockers (valsartan and candesartan), a loop diuretic (torasemide), and an aldosterone inhibitor (spironolactone). Similar results were obtained by other investigators, such as Somsen et al. (20) (for enalapril) and Lotze et al. (21) and de Milliano et al. (22) (for various $\beta$ blockers).

${ }^{123}$ I-MIBG SPECT has been shown to be complementary to planar imaging in studies of coronary artery disease after an acute myocardial infarction (MI) $(23,24)$. Viable but denervated myocardium has been shown to be supersensitive to the effects of infused catecholamines (25), which may provide a substrate for the genesis of ventricular arrhythmias. For this reason, the results of ${ }^{123}$ I-MIBG SPECT and MPI SPECT are often compared by identifying segments with adrenergic/perfusion mismatches. As in MPI SPECT studies, the myocardium is usually subdivided into several segments (ranging from 5 to 20), and each location is scored for severity (on scales from 0 to 3 or 0 to 4 ).

Three categories of ${ }^{123}$ I-MIBG SPECT and MPI SPECT findings are commonly reported: both normal; both equivalently abnormal (matched defects); or ${ }^{123}$ I-MIBG SPECT defect more severe than MPI SPECT defect (mismatched defects) (Fig. 2). A defect less severe on ${ }^{123}$ I-MIBG SPECT than on MPI SPECT is rarely, if ever, observed. Mismatched defects may be subcategorized in terms of whether the area in question demonstrates ischemia on stress MPI or remains unchanged.

Most studies of ${ }^{123}$ I-MIBG imaging in patients after MI have compared defects on ${ }^{123}$ I-MIBG SPECT with results on rest and stress MPI to assess the relationship between areas of infarction and denervation. Three small studies (26-28) in which 58 patients were examined within 3 mo after MI arrived at the joint conclusion that the ${ }^{123}$ I-MIBG defects were generally larger than those on MPI, whether determined from quantitative count-based assessments or from semiquantitative scoring of SPECT myocardial segments. Given that sympathetic nerve fibers in the heart travel in the subepicardium parallel to the vascular structures and penetrate the underlying myocardium, it is conceivable that this difference in defect size, among others, is caused by the fact that neural tissue has a greater sensitivity to hypoxia than myocardial fibers and has a longer recovery time $(29,30)$. More recent studies, such as those by Simões et al. (31), Marini et al. (32), and Hayashi et al. (33), confirmed the presence of denervated areas extending beyond the infarct borders and proposed associations with depolarization abnormalities, unappreciated myocardial necrosis, and long-term variability in induced ventricular tachyarrhythmias.

Interest has been shown in the potential for the global quantitation of ${ }^{123}$ I-MIBG SPECT images to replace the planar HMR for diagnostic and prognostic purposes. An analysis of the ${ }^{123}$ I-MIBG SPECT studies from the AdreView Myocardial Imaging for Risk Evaluation in Heart Failure (ADMIRE-HF) trial, which is a prospective trial evaluating ${ }^{123} \mathrm{I}-\mathrm{MIBG}$ imaging for identifying patients who have HF and are most likely to experience cardiac events, demonstrated that the SPECT HMR was equivalent to the planar HMR for discriminating between patients with $\mathrm{HF}$ and control subjects $(6,34)$. Additionally, the ability of ${ }^{123} \mathrm{I}-\mathrm{MIBG}$ SPECT to provide regional information not available on planar images remains a driver for efforts to incorporate this procedure into assessments of patients with HF for arrhythmic event risk (35).

\section{I-MIBG SPECT FOR ARRHYTHMIC EVENT RISK ASSESSMENT}

Better arrhythmic event risk assessment is necessary given that in the MADIT II trial, only $23.5 \%$ of the 720 patients with HF and a prophylactic implantable cardioverter defibrillator (ICD) received antiarrhythmia device therapy for ventricular tachyarrhythmia (36). Early research on experimentally denervated myocardium suggested that such regions were much more sensitive to norepinephrine infusion than normally innervated regions, a situation considered potentially proarrhythmic (37). Over the subsequent decades, ${ }^{123}$ I-MIBG SPECT was used in numerous studies to document such regional denervation and its association with naturally occurring or inducible ventricular arrhythmic events. The dominant observation was that the larger the extent of the ${ }^{123}$ I-MIBG SPECT abnormality, the higher the likelihood of ventricular tachyarrhythmia (38-41).

Most investigators performing ${ }^{123}$ I-MIBG SPECT studies had expected to find that arrhythmic event risk would increase with the size of the denervated area as well as the amount of innervationperfusion mismatch, as reflected by paired ${ }^{123}$ I-MIBG and MPI SPECT studies. Another expectation was that there would be a dividing point in ${ }^{123} \mathrm{I}-\mathrm{MIBG}$ defect size between patients with "low risk" and patients with "high risk" for arrhythmic events. In the small study by Arora et al. (38), both mean ${ }^{123}$ I-MIBG defect scores and the number of late mismatches were higher in the 10 patients who had experienced appropriate ICD discharges than in the 7 who had not. Patients who had ischemic heart disease and inducible ventricular tachycardia on electrophysiology testing had 


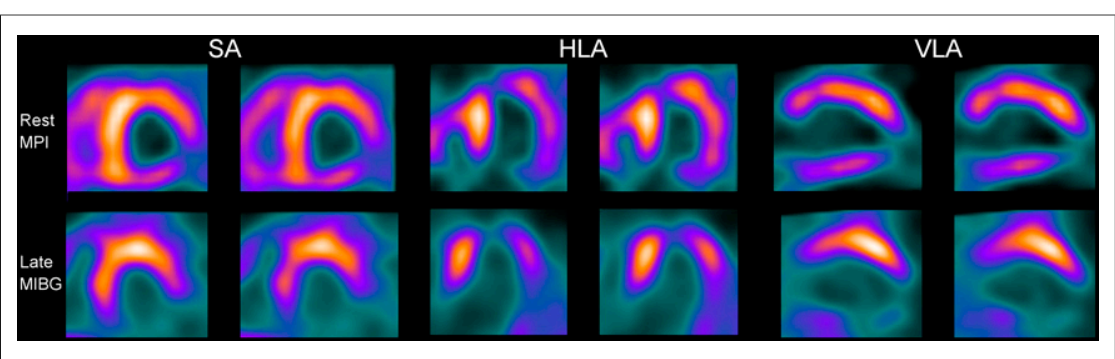

FIGURE 2. Innervation-perfusion mismatch between ${ }^{123}$ I-MIBG SPECT and rest MPI with 99mTc-tetrofosmin. Rest MPI demonstrated large inferoposterolateral expanding apical defect in 63-y-old patient with ischemic cardiomyopathy. ${ }^{123}$ I-MIBG SPECT demonstrated more extended inferolateral defect. HLA = horizontal axis; SA = short axis; VLA = vertical long axis. studies as normal, matched, or mismatched resulted in similar findings; patients with $\mathrm{HF}$ and 1 or 2 mismatched regions had the highest arrhythmic event rates (46). In both of those analyses, the largest absolute number of arrhythmic events was seen in patients with the most severe defects, but the highest proportion was seen in patients with intermediateseverity defects.

The aforementioned results attest to the mechanistic complexity of arrhythmia generation and support the conclusion that no single test can identify all at-risk patients with HF. Nevertheless, there is growing evidence that ${ }^{123}$ I-MIBG SPECT imaging higher total ${ }^{123}$ I-MIBG SPECT defect scores than patients who did not have inducible ventricular tachycardia, with a late total defect score threshold of 37 , yielding a $77 \%$ sensitivity and a $75 \%$ specificity (39). In the prospective study by Boogers et al. (40), patients with an ICD and a late total defect score of greater than 26 were 10 times more likely to receive appropriate ICD therapy (cumulative 3-y event rate: $52 \%$ vs. $5 \% ; P<0.01$ ). Interestingly, in the latter 2 studies, ${ }^{123} \mathrm{I}$ MIBG SPECT defect size was a significant predictor of arrhythmic events but not mismatch scores. In contrast, Marshall et al. (42) found differences in both the total ${ }^{123}$ I-MIBG SPECT defect score $(37.0 \pm$ 9.4 [mean $\pm \mathrm{SD}$ ] vs. $25.5 \pm 7.7 ; P=0.001)$ and the mismatch score $(18.5 \pm 8.5$ vs. $8.4 \pm 5.0 ; P<0.01)$ between patients with ICD firing and those without ICD firing. As in the study of Bax et al. (39), a total ${ }^{123}$ I-MIBG SPECT defect score of greater than or equal to 31 had a sensitivity of $78 \%$ and a specificity of $77 \%$.

Recent evidence suggests that quantitative characterization of myocardial transition zones (between normal and infarcted or denervated myocardium) may supplement the more simple definition of innervation-perfusion mismatch. In a quantitative reanalysis of the SPECT images that were interpreted visually in the study of Bax et al. (39), MPI scar extent, border zone extent, and ${ }^{123}$ I-MIBG uptake in the border zone were analyzed; the best prediction accuracy for ventricular tachycardia inducibility was achieved with the last (area under the receiver operating characteristic curve, 0.78) (43). In a prospective study of 15 patients referred for ischemic ventricular tachycardia ablation, 3-dimensional innervation models were derived from ${ }^{123} \mathrm{I}$ MIBG SPECT examinations and registered to high-density voltage maps (44). ${ }^{123}$ I-MIBG innervation defects were approximately 2.5fold larger than bipolar voltage-defined scars, all ventricular tachycardia ablation sites were within areas of abnormal innervation, but $36 \%$ of successful ablation sites demonstrated normal voltages $(>1.5 \mathrm{mV})$. These studies strongly suggested that innovations in quantitative analysis techniques represent the future of ${ }^{123}$ I-MIBG SPECT for identifying arrhythmic event risk and guiding therapy.

Studies have also raised questions about the relationship between ${ }^{123}$ I-MIBG defect severity and arrhythmic event risk. Planar HMR data showed that the highest occurrence of arrhythmic events was seen in patients with $\mathrm{HF}$ and an intermediate reduction in uptake rather than the lowest values. In 2014, Verberne et al. presented the results of an informed reinterpretation of ${ }^{123}$ I-MIBG and MPI SPECT studies from the ADMIRE-HF trial; the results showed that patients with HF and an intermediate severity of total defect scores (14-28 of a possible maximum of 68) had higher arrhythmic event rates than those with more severe defects (45). As part of the same study, visual scoring of regions (anterior, inferior, septal, lateral, and apical) on ${ }^{123}$ I-MIBG and MPI SPECT should be considered for the assessment of patients with HF for appropriate therapeutic interventions.

\section{ADVANCES IN ${ }^{123}$ |-MIBG SPECT QUANTITATION}

${ }^{123}$ I-MIBG is internalized by neuroendocrine cells through the rapid energy-dependent uptake mechanism (47), reaching a relative plateau within a few minutes after injection. It is stored, unmetabolized, in the neurosecretory granules, resulting in a specific concentration, unlike in cells of other tissues. Because of flow-limited uptake of the ${ }^{123}$ I-MIBG tracer, retention measures are insensitive to low to moderate levels of regional denervation and decline only when nerve losses become fairly severe (48).

Since the earliest investigations of ${ }^{123} \mathrm{I}-\mathrm{MIBG}$ SPECT in cardiology, quantitation of the size and the severity of innervation abnormalities has been performed (49). Initially, semiquantitative scoring from a visual analysis, analogous to that used in MPI, was performed, as noted earlier for studies of patients after MI $(27,28)$. When quantitative bull's-eye mapping for MPI SPECT became readily available, it was also applied to ${ }^{123} \mathrm{I}$-MIBG SPECT analysis $(23,50-52)$. However, because the quantitative programs were usually adapted from those developed for MPI SPECT, they did not take into account the unique physical properties of MIBG iodinated with ${ }^{123} \mathrm{I}$, which affects image quality. These include the presence of a significant number of high-energy photons (1.1\% yield of 529-keV $\gamma$ rays) (53); accumulation in the liver that overlaps the inferior wall (Fig. 3); and scattering from the lung field to the lateral left ventricular wall (54), as a result of which the normal cardiac ${ }^{123}$ I-MIBG distribution includes relatively low uptake in the inferior wall (55), which is more pronounced in the elderly. Therefore, in contrast to what is observed in PET examinations with ${ }^{11} \mathrm{C}$-hydroxyephedrine and ${ }^{18} \mathrm{~F}$-fluorodopamine, the regional ${ }^{123} \mathrm{I}$ MIBG uptake in the left ventricle is heterogeneous in healthy subjects, as observed by Morozumi et al. (56) and Yoshinaga et al. (57).

More recently, programs and protocols have been developed specifically for ${ }^{123} \mathrm{I}$-labeled compounds such as ${ }^{123} \mathrm{I}-\mathrm{MIBG}$. These have included the introduction of iterative reconstruction techniques with compensation for scatter and septal penetration $(53,58,59)$ and the use of volumetric analysis techniques (60). The development of ${ }^{123}$ I-MIBG databases for healthy subjects has also provided a more reliable means for quantifying the significance of reduced uptake in $\mathrm{HF}$ and other cardiology patients (6).

The potential of improved ${ }^{123}$ I-MIBG quantitation techniques has been explored in recent reevaluations of the large ${ }^{123} \mathrm{I}-\mathrm{MIBG}$ SPECT imaging database from the ADMIRE-HF trial. Through the use of a variable reference threshold for pixel-based abnormalities based on the planar HMR, quantitative differences between ${ }^{123} \mathrm{I}-\mathrm{MIBG}$ 


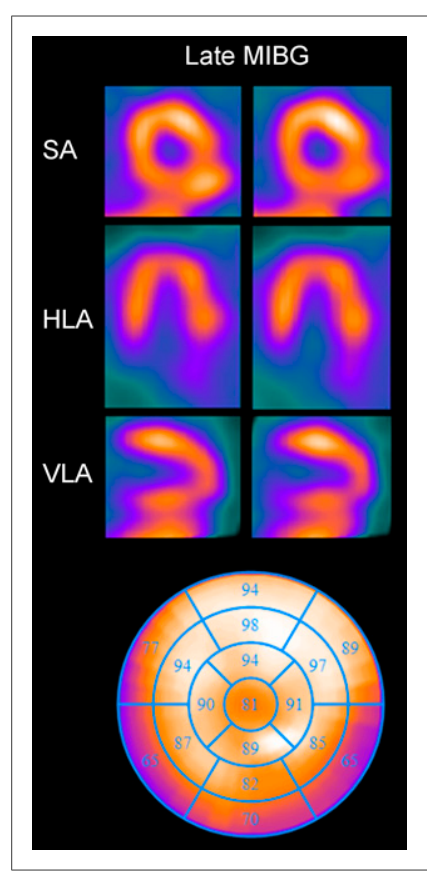

FIGURE 3. ${ }^{123}$ |-MIBG SPECT images in short axis (SA), horizontal axis (HLA), vertical long axis (VLA), and bull's-eye demonstrate that accumulation of ${ }^{123 \mid}$-MIBG in liver and bowel overlaps inferior wall and affects image quality. MIBG protocol (63). Nevertheless, it can be challenging to evaluate ${ }^{123}$ I-MIBG SPECT studies visually, especially in patients with $\mathrm{HF}$ and a global reduction in uptake (35). Automated quantitation of ${ }^{123}$ I-MIBG SPECT studies offers the potential to overcome some of the limitations of visual image interpretation and improves the clinical utility of such studies for diagnosis and prognosis.

\section{3|-MIBG SPECT WITH SOLID-STATE CAMERAS}

The recent introduction of new solid-state cardiac nuclear cameras that provide $180^{\circ}$ of imaging without the need for gantry rotation, improved collimator design, and optimized acquisition geometry with increased photon sensitivity and contrast resolution has created the potential for more rapid SPECT acquisitions or a reduction in radiopharmaceutical activity and thus a lower dose for patients (64-66).

This technology also offers advantages for ${ }^{123}$ I-MIBG SPECT imaging, including better energy discrimination and temporal resolution (67). List-mode acquisition analogous to that used in PET presents an opportunity to obtain true dynamic SPECT data, which could provide new physiologic insights to enhance the information content of the examination.

Initial experiences with ${ }^{123} \mathrm{I}$-MIBG SPECT imaging and solidstate cadmium-zinc-telluride cameras have been promising. Patients with left ventricular dysfunction were shown to have higher ${ }^{123} \mathrm{I}$ MIBG summed defect scores than those with normal function (68). Similar results were obtained when mechanical dyssynchrony on MPI SPECT was compared with ${ }^{123}$ I-MIBG SPECT findings (69).

Data on the in vivo myocardial kinetics of ${ }^{123} \mathrm{I}-\mathrm{MIBG}$ are rare. Dynamic ${ }^{123}$ I-MIBG SPECT has the potential for defining new quan- titative parameters, as shown in early human experiments. Tinti et al. demonstrated the feasibility of dynamic 3-dimensional ${ }^{123} \mathrm{I}-\mathrm{MIBG}$ kinetic analysis for providing additional information on cardiac innervation, particularly through the analysis of time-activity curves for ${ }^{123}$ I-MIBG (70). Also, in an experimental pig model, myocardial peak uptake was observed earlier than had been previously described (71).

Although the possibilities of dynamic ${ }^{123}$ I-MIBG SPECT were initially explored with conventional rotating $\gamma$ cameras (72), the clinical potential of this technique with cadmium-zinc-telluride cameras likely will be established as the new solid-state camera technology matures and more users gain experience with it.

\section{COMPARISON OF SPECT AND PET TECHNIQUES}

The superior quantitative capabilities of PET imaging make PET an attractive alternative to SPECT. However, unlike compounds such as ${ }^{123} \mathrm{I}-\mathrm{MIBG}$, which can be centrally manufactured and widely distributed commercially, most PET agents are labeled with short-half-life isotopes such as ${ }^{11} \mathrm{C}$ and therefore are available only in facilities with an on-site cyclotron. Nevertheless, as a research tool, PET remains extremely valuable. The PET agent most analogous to ${ }^{123}$ I-MIBG is metahydroxyephedrine (mHED), as the uptake of both by sympathetic neurons is mediated by the norepinephrine transporter. However, in only a few studies has the cardiac uptake of ${ }^{123} \mathrm{I}-\mathrm{MIBG}$ been directly compared with that of mHED.

In a study of rabbits injected with both ${ }^{123}$ I-MIBG and mHED, similar reductions in uptake in denervated myocardium were observed (73). However, pretreatment with the norepinephrinedepleting compound reserpine had a greater effect on mHED uptake than on ${ }^{123}$ I-MIBG uptake, suggesting that the former might be more specific for intravesicular uptake than the latter. Luisi et al. showed that the relative retention of $\mathrm{mHED}$ was significantly greater than that of ${ }^{123} \mathrm{I}-\mathrm{MIBG}$ in pigs, reflecting improved specificity as a result of less nonspecific uptake of the former tracer (74). Rischpler et al. (75) reported that the ${ }^{131} \mathrm{I}-\mathrm{MIBG}$ uptake defect in rats matched the area of an earlier MI, whereas the mHED uptake defect was larger. However, in a direct comparison of ${ }^{123} \mathrm{I}-\mathrm{MIBG}$ SPECT and mHED PET in 21 patients with left ventricular dysfunction, Matsunari et al. (76) reported a high correlation between defect sizes in the 2 methods; however, late ${ }^{123}$ I-MIBG SPECT overestimated defect sizes in the inferior and septal regions, presumably because of image quality issues caused by adjacent liver activity.

Although the early development of an ${ }^{18} \mathrm{~F}$-labeled compound for PET imaging of sympathetic neurons is continuing (77), for the foreseeable future ${ }^{123} \mathrm{I}-\mathrm{MIBG}$ SPECT will remain the only widely available nuclear imaging method for assessing regional myocardial sympathetic innervation.

\section{CONCLUSION}

The shift from planar to SPECT techniques in ${ }^{123} \mathrm{I}-\mathrm{MIBG}$ imaging has been slow in comparison with what occurred in clinical MPI 25-30 y ago. Nevertheless, the accumulation of evidence regarding the value of ${ }^{123}$ I-MIBG SPECT results, along with improvements in imaging equipment and image processing techniques, should result in acceleration of the growth and clinical use of the technique in the coming years.

\section{DISCLOSURE}

No potential conflict of interest relevant to this article was reported. 


\section{REFERENCES}

1. Wieland DM, Wu J, Brown LE, Mangner TJ, Swanson DP, Beierwaltes WH. Radiolabeled adrenergic neuron-blocking agents: adrenomedullary imaging with 131 iodobenzylguanidine. J Nucl Med. 1980;21:349-353.

2. Yamashina S, Yamazaki J. Neuronal imaging using SPECT. Eur J Nucl Med Mol Imaging. 2007;34:939-950.

3. Verberne HJ, Brewster LM, Somsen GA, Eck-Smit BL. Prognostic value of myocardial ${ }^{123}$ I-metaiodobenzylguanidine (MIBG) parameters in patients with heart failure: a systematic review. Eur Heart J. 2008;29:1147-1159.

4. Tanaka T, Aizawa T, Kato K, et al. Estimation of regional myocardial sympathetic neuronal function with I-123 metaiodobenzylguanidine (MIBG) myocardial images in patients with cardiomyopathy. Kaku Igaku. 1989;26:257-261.

5. Nakajima K, Bunko H, Taki J, Shimizu M, Muramori A, Hisada K. Quantitative analysis of ${ }^{123}$ I-meta-iodobenzylguanidine (MIBG) uptake in hypertrophic cardiomyopathy. Am Heart J. 1990;119:1329-1337.

6. Chen J, Folks RD, Verdes L, Manatunga DN, Jacobson AF, Garcia EV. Quantitative I-123 mIBG SPECT in differentiating abnormal and normal mIBG myocardial uptake. J Nucl Cardiol. 2012;19:92-99.

7. Clements IP, Garcia EV, Chen J, Folks RD, Butler J, Jacobson AF. Quantitative iodine-123-metaiodobenzylguanidine (MIBG) SPECT imaging in heart failure with left ventricular systolic dysfunction: development and validation of automated procedures in conjunction with technetium-99m tetrofosmin myocardial perfusion SPECT. J Nucl Cardiol. March 19, 2015 [Epub ahead of print].

8. Somsen GA, Verberne HJ, Fleury E, Righetti A. Normal values and within subject variability of cardiac I-123 MIBG scintigraphy in healthy individuals: implications of clinical studies. J Nucl Cardiol. 2004;11:126-133.

9. Tsukamoto M, Terada K, Yoneyama S, Tatsukawa H, Katoh S. Evaluation of ${ }^{123}$ I-MIBG clearance from the myocardium: comparison of two methodsSPECT and planar methods [in Japanese]. Kaku Igaku. 1997;34:827-830.

10. Savarese G, Losco T, Parente A, et al. Clinical applications of MIBG SPECT in chronic heart failure [in Italian]. G Ital Cardiol (Rome). 2012;13:91-97.

11. Kuwabara Y, Tamaki N, Nakata T, et al. Determination of the survival rate in patients with congestive heart failure stratified by ${ }^{123}$ I-MIBG imaging: a metaanalysis from the studies performed in Japan. Ann Nucl Med. 2011;25:101-107.

12. Kasama S, Toyama T, Kumakura H, et al. Spironolactone improves cardiac sympathetic nerve activity and symptoms in patients with congestive heart failure. J Nucl Med. 2002;43:1279-1285.

13. Kasama S, Toyama T, Kumakura H, et al. Addition of valsartan to an angiotensinconverting enzyme inhibitor improves cardiac sympathetic nerve activity and left ventricular function in patients with congestive heart failure. J Nucl Med. 2003;44:884-890.

14. Kasama S, Toyama T, Kumakura H, et al. Effects of perindopril on cardiac sympathetic nerve activity in patients with congestive heart failure: comparison with enalapril. Eur J Nucl Med Mol Imaging. 2005;32:964-971.

15. Kasama S, Toyama T, Kumakura H, et al. Effects of candesartan on cardiac sympathetic nerve activity in patients with congestive heart failure and preserved left ventricular ejection fraction. J Am Coll Cardiol. 2005;45:661-667.

16. Kasama S, Toyama T, Hatori $\mathrm{T}$, et al. Effects of torasemide on cardiac sympathetic nerve activity and left ventricular remodeling in patients with congestive heart failure. Heart. 2006;92:1434-1440.

17. Kasama S, Toyama T, Hatori $\mathrm{T}$, et al. Comparative effects of valsartan and enalapril on cardiac sympathetic nerve activity and plasma brain natriuretic peptide in patients with congestive heart failure. Heart. 2006;92:625-630.

18. Kasama S, Toyama T, Sumino H, et al. Additive effects of spironolactone and candesartan on cardiac sympathetic nerve activity and left ventricular remodeling in patients with congestive heart failure. J Nucl Med. 2007;48:1993-2000.

19. Kasama S, Toyama T, Iwasaki T, et al. Evaluation of cardiac sympathetic nerve activity and aldosterone suppression in patients with acute decompensated heart failure on treatment containing intravenous atrial natriuretic peptide. Eur J Nucl Med Mol Imaging. 2014;41:1683-1691.

20. Somsen GA, van Vlies B, de Milliano PA, et al. Increased myocardial ${ }^{123}$ Imetaiodobenzylguanidine uptake after enalapril treatment in patients with chronic heart failure. Heart. 1996;76:218-222.

21. Lotze U, Kaepplinger S, Kober A, Richartz BM, Gottschild D, Figulla HR. Recovery of the cardiac adrenergic nervous system after long-term beta-blocker therapy in idiopathic dilated cardiomyopathy: assessment by increase in myocardial ${ }^{123}$ I-metaiodobenzylguanidine. J Nucl Med. 2001;42:49-54.

22. de Milliano PA, de Groot AC, Tijssen JG, van Eck-Smit BL, van Zwieten PA, Lie KI. Beneficial effects of metoprolol on myocardial sympathetic function: evidence from a randomized, placebo-controlled study in patients with congestive heart failure [abstract]. Am Heart J. 2002;144:A14-A22.

23. Matsunari I, Schricke U, Bengel FM, et al. Extent of cardiac sympathetic neuronal damage is determined by the area of ischemia in patients with acute coronary syndromes. Circulation. 2000;101:2579-2585.
24. Estorch M, Narula J, Flotats A, et al. Concordance between rest MIBG and exercise tetrofosmin defects: possible use of rest MIBG imaging as a marker of reversible ischaemia. Eur J Nucl Med. 2001;28:614-619.

25. Kammerling JJ, Green FJ, Watanabe AM, et al. Denervation supersensitivity of refractoriness in noninfarcted areas apical to transmural myocardial infarction. Circulation. 1987;76:383-393.

26. Estorch M, Flotats A, Serra-Grima R, et al. Influence of exercise rehabilitation on myocardial perfusion and sympathetic heart innervation in ischaemic heart disease. Eur J Nucl Med. 2000;27:333-339.

27. Hartikainen J, Mantysaari M, Kuikka J, et al. Extent of cardiac autonomic denervation in relation to angina on exercise test in patients with recent acute myocardial infarction. Am J Cardiol. 1994;74:760-763.

28. McGhie AI, Corbett JR, Akers MS, et al. Regional cardiac adrenergic function using I-123 meta-iodobenzylguanidine tomographic imaging after acute myocardial infarction. Am J Cardiol. 1991;67:236-242.

29. Dae MW, O'Connell JW, Botvinick EH, et al. Acute and chronic effects of transient myocardial ischemia on sympathetic nerve activity, density, and norepinephrine content. Cardiovasc Res. 1995;30:270-280.

30. Barber MJ, Mueller TM, Henry DP, Felten SY, Zipes DP. Transmural myocardial infarction in the dog produces sympathectomy in noninfarcted myocardium. Circulation. 1983;67:787-796.

31. Simões MV, Barthel P, Matsunari I, et al. Presence of sympathetically denervated but viable myocardium and its electrophysiologic correlates after early revascularised, acute myocardial infarction. Eur Heart J. 2004;25:551-557.

32. Marini C, Giorgetti A, Gimelli A, et al. Extension of myocardial necrosis differently affects MIBG retention in heart failure caused by ischaemic heart disease or by dilated cardiomyopathy. Eur J Nucl Med Mol Imaging. 2005;32:682-688.

33. Hayashi M, Kobayashi Y, Morita N, et al. Clinical significance and contributing factors of long-term variability in induced ventricular tachyarrhythmias. J Cardiovasc Electrophysiol. 2003;14:1049-1056.

34. Jacobson AF, Senior R, Cerqueria MD, et al. Myocardial iodine-123 metaiodobenzylguanidine imaging and cardiac events in heart failure: results of the prospective ADMIRE-HF (AdreView Myocardial Imaging for Risk Evaluation in Heart Failure) study. J Am Coll Cardiol. 2010;55:2212-2221.

35. Travin MI. Cardiac radionuclide imaging to assess patients with heart failure. Semin Nucl Med. 2014;44:294-313.

36. Moss AJ, Greenberg H, Case RB, et al. Long-term clinical course of patients after termination of ventricular tachyarrhythmia by an implanted defibrillator. Circulation. 2004;110:3760-3765.

37. Stanton MS, Zipes DP. Modulation of drug effects by regional sympathetic denervation and supersensitivity. Circulation. 1991;84:1709-1714.

38. Arora R, Ferrick KJ, Nakata T, et al. I-123 MIBG imaging and heart rate variability analysis to predict the need for an implantable cardioverter defibrillator. J Nucl Cardiol. 2003;10:121-131.

39. Bax JJ, Kraft O, Buxton AE, et al. 123 I-mIBG scintigraphy to predict inducibility of ventricular arrhythmias on cardiac electrophysiology testing: a prospective multicenter pilot study. Circ Cardiovasc Imaging. 2008;1:131-140.

40. Boogers MJ, Borleffs CJW, Henneman MM, et al. Cardiac sympathetic denervation assessed with 123-iodine metaiodobenzylguanidine imaging predicts ventricular arrhythmias in implantable cardioverter-defibrillator patients. J Am Coll Cardiol. 2010;55:2769-2777.

41. Miranda CH, Figueiredo AB, Maciel BC, Marin-Neto JA, Simões MV. Sustained ventricular tachycardia is associated with regional myocardial sympathetic denervation assessed with ${ }^{123} \mathrm{I}$-metaiodobenzylguanidine in chronic Chagas cardiomyopathy. J Nucl Med. 2011;52:504-510.

42. Marshall A, Cheetham A, George RS, Mason M, Kelion AD. Cardiac iodine-123 metaiodobenzylguanidine imaging predicts ventricular arrhythmia in heart failure patients receiving an implantable cardioverter-defibrillator for primary prevention. Heart. 2012;98:1359-1365.

43. Zhou Y, Zhou W, Folks RD, et al. I-123 mIBG and Tc-99m myocardial SPECT imaging to predict inducibility of ventricular arrhythmia on electrophysiology testing: a retrospective analysis. J Nucl Cardiol. 2014;21:913-920.

44. Klein T, Abdulghani M, Smith M, et al. Three-dimensional ${ }^{123}$ I-meta-iodobenzylguanidine cardiac innervation maps to assess substrate and successful ablation sites for ventricular tachycardia: a feasibility study for a novel paradigm of innervation imaging. Circ Arrhythm Electrophysiol. February 23, 2015 [Epub ahead of print].

45. Verberne H, Henzlova M, Jain D, et al. ${ }^{123} \mathrm{I}-\mathrm{mIBG}$ and ${ }^{99 \mathrm{~m}}$ Tc-tetrofosmin SPECT for prediction of arrhythmic risk in ischemic heart failure patients [abstract]. J Nucl Med. 2014;55(suppl 1):182.

46. Verberne HJ, Henzlova MJ, Jain D, et al. Regional myocardial mismatch between ${ }^{123} \mathrm{I}$-mIBG and ${ }^{99 \mathrm{~m}} \mathrm{Tc}$-tetrofosmin SPECT for the prediction of arrhythmic events in ischemic heart failure patients [abstract]. J Nucl Cardiol. 2014;21:799. 
47. Nakajima K, Okuda K, Matsuo S, et al. Standardization of metaiodobenzylguanidine heart to mediastinum ratio using a calibration phantom: effects of correction on normal databases and a multicentre study. Eur J Nucl Med Mol Imaging. 2012;39:113-119.

48. Raffel DM. Targeting norepinephrine transporters in cardiac sympathetic nerve terminals. In: Welch MJ, Eckelman WC, eds. Targeted Molecular Imaging. Boca Raton, FL: CRC Press; 2012:305-320.

49. Hirosawa K, Tanaka T, Hisada K, Bunko H. Clinical evaluation of ${ }^{123}$ I-MIBG for assessment of the sympathetic nervous system in the heart (multi-center clinical trial) [in Japanese]. Kaku Igaku. 1991;28:461-476.

50. Lekakis J, Antoniou A, Vassilopoulos N, et al. I-123 metaiodobenzylguanidinethallium-201 mismatch following myocardial infarction. Clin Cardiol. 1994; $17: 21-25$.

51. Inobe $\mathrm{Y}$, Kugiyama K, Miyagi H, et al. Long-lasting abnormalities in cardiac sympathetic nervous system in patients with coronary spastic angina: quantitative analysis with iodine 123 metaiodobenzylguanidine myocardial scintigraphy. Am Heart J. 1997;134:112-118.

52. Garcia EV, Faber TL, Cooke CD, Folks RD, Chen J, Santana C. The increasing role of quantification in nuclear cardiology: the Emory approach. J Nucl Cardiol. 2007; 14:420-432.

53. Chen J, Garcia EV, Galt JR, Folks RD, Carrio I. Improved quantification in 123-I cardiac SPECT imaging with deconvolution of septal penetration. Nucl Med Commun. 2006;27:551-558.

54. Verberne HJ, Somsen GA, Povinec P, van Eck-Smit BL, Jacobson AF. Impact of mediastinal, liver and lung ${ }^{123} \mathrm{I}$-metaiodobenzylguanidine ( $\left.{ }^{123} \mathrm{I}-\mathrm{MIBG}\right)$ washout on calculated ${ }^{123}$ I-MIBG myocardial washout. Eur J Nucl Med Mol Imaging. 2009;36:1322-1328.

55. Gill JS, Hunter GJ, Gane G, Camm AJ. Heterogeneity of the human myocardial sympathetic innervation: in vivo demonstration by iodine 123-labeled metaiodobenzylguanidine scintigraphy. Am Heart J. 1993;126:390-398.

56. Morozumi T, Kusuoka H, Fukuchi K, et al. Myocardial iodine-123-metaiodobenzylguanidine images and autonomic nerve activity in normal subjects. $\mathrm{J} \mathrm{Nucl}$ Med. 1997;38:49-52.

57. Yoshinaga K, Tomiyama Y, Manabe O, et al. Prone-position acquisition of myocardial ${ }^{123}$ I-metaiodobenzylguanidine (MIBG) SPECT reveals regional uptake similar to that found using ${ }^{11} \mathrm{C}$-hydroxyephedrine PET/CT. Ann Nucl Med. 2014;28:761-769.

58. Motomura N, Ichihara T, Takayama T, Aoki S, Kubo H, Takeda K. Practical compensation method of downscattered component due to high energy photon in 123I imaging [in Japanese]. Kaku Igaku. 1999;36:997-1005.

59. Chen J, Garcia EV, Galt JR, Folks RD, Carrio I. Optimized acquisition and processing protocols for I-123 cardiac SPECT imaging. J Nucl Cardiol. 2006;13:251-260.

60. van der Veen BJ, Younis IA, de Roos A, Stokkel MPM. Assessment of global cardiac I-123 MIBG uptake and washout using volumetric quantification of SPECT acquisitions. J Nucl Cardiol. 2012;19:752-762.

61. Clements IP, Wiseman GA, Hodge DO. Differences in myocardial [ $\left.{ }^{123} \mathrm{I}\right]-\mathrm{mIBG}$ uptake in ischemic and non-ischemic cardiomyopathy [abstract]. Eur J Nucl Med Mol Imaging. 2012;39:S192.
62. Clements IP, Garcia EV, Folks RD, Butler J, Jacobson AF. Differences in myocardial sympathetic innervation and perfusion in patients with ischemic versus non-ischemic heart failure. J Card Fail. 2014;20(suppl):S17.

63. Pellegrino T, Petretta M, De Luca S, et al. Observer reproducibility of results from a low-dose ${ }^{123}$ I-metaiodobenzylguanidine cardiac imaging protocol in patients with heart failure. Eur J Nucl Med Mol Imaging. 2013;40:1549-1557.

64. Case JA, Bateman TM. Taking the perfect nuclear image: quality control, acquisition, and processing techniques for cardiac SPECT, PET, and hybrid imaging. J Nucl Cardiol. 2013;20:891-907.

65. Esteves FP, Raggi P, Folks RD, et al. Novel solid-state-detector dedicated cardiac camera for fast myocardial perfusion imaging: multicenter comparison with standard dual detector camera. J Nucl Cardiol. 2009;16:927-934.

66. Duvall WL, Croft LB, Ginsberg ES, et al. Reduced isotope dose and imaging time with a high-efficiency CZT SPECT camera. J Nucl Cardiol. 2011;18:847857.

67. Garcia EV. Physical attributes, limitations, and future potential for PET and SPECT. J Nucl Cardiol. 2012;19(suppl):S19-S29.

68. Gimelli A, Liga R, Giorgetti A, Genovesi D, Marzullo P. Assessment of myocardial adrenergic innervation with a solid-state dedicated cardiac cadmiumzinc-telluride camera: first clinical experience. Eur Heart J Cardiovasc Imaging. 2014;15:575-585.

69. Gimelli A, Liga R, Genovesi D, Giorgetti A, Kusch A, Marzullo P. Association between left ventricular regional sympathetic denervation and mechanical dyssynchrony in phase analysis: a cardiac CZT study. Eur J Nucl Med Mol Imaging. 2014;41:946-955.

70. Tinti E, Positano V, Giorgetti A, Marzullo P. Feasibility of ${ }^{123}$ I-meta-iodobenzylguanidine dynamic 3-D kinetic analysis in vivo using a CZT ultrafast camera: preliminary results. Eur J Nucl Med Mol Imaging. 2014;41:167-173.

71. Giorgetti A, Burchielli S, Positano V, et al. Dynamic 3D analysis of myocardial sympathetic innervation: an experimental study using ${ }^{123} \mathrm{I}-\mathrm{MIBG}$ and a CZT camera. J Nucl Med. 2015;56:464-469.

72. Arimoto T, Takeishi Y, Fukui A, et al. Dynamic ${ }^{123}$ I-MIBG SPECT reflects sympathetic nervous integrity and predicts clinical outcome in patients with chronic heart failure. Ann Nucl Med. 2004;18:145-150.

73. Nomura Y, Matsunari I, Takamatsu H, et al. Quantitation of cardiac sympathetic innervation in rabbits using ${ }^{11} \mathrm{C}$-hydroxyephedrine PET: relation to ${ }^{123} \mathrm{I}-\mathrm{MIBG}$ uptake. Eur J Nucl Med Mol Imaging. 2006;33:871-878.

74. Luisi AJ Jr, Suzuki G, Dekemp R, et al. Regional ${ }^{11} \mathrm{C}$-hydroxyephedrine retention in hibernating myocardium: chronic inhomogeneity of sympathetic innervation in the absence of infarction. $J$ Nucl Med. 2005;46:1368-1374.

75. Rischpler C, Fukushima K, Isoda T, et al. Discrepant uptake of the radiolabeled norepinephrine analogues hydroxyephedrine (HED) and metaiodobenzylguanidine (MIBG) in rat hearts. Eur J Nucl Med Mol Imaging. 2013;40:1077-1083.

76. Matsunari I, Aoki H, Nomura Y, et al. Iodine-123 metaiodobenzylguanidine imaging and carbon-11 hydroxyephedrine positron emission tomography compared in patients with left ventricular dysfunction. Circ Cardiovasc Imaging. 2010;3:595-603.

77. Sinusas AJ, Lazewatsky J, Brunetti J, et al. Biodistribution and radiation dosimetry of LMI1195: first-in-human study of a novel ${ }^{18} \mathrm{~F}$-labeled tracer for imaging myocardial innervation. J Nucl Med. 2014;55:1445-1451. 